\title{
ON POLYTOPES WITH SMALL FACES
}

\author{
by J. N. LILLINGTON
}

(Received 18 July, 1975)

Introduction. In this paper all sets considered are assumed to be compact subsets of Euclidean Space $E^{n}$. A number of results concerning the total edge-lengths of polyhedra have been given by various authors, many of which are mentioned in references in [1]. In [1], it was conjectured that all polytopes inscribed in the unit sphere and containing its centre have total edge-length greater than $2 n$. This was proved true for simplicial polytopes and shown to be best possible in the sense that there exist simplices with the stated property and with total edge-length arbitrarily close to $2 n$. In this paper we shall show that the bound is not always best possible if the magnitudes of the faces of such polytopes are restricted and we shall also give some related results on surface areas. This work was carried out while the author was a research student at Royal Holloway College, London and is a revised version of part of the author's thesis approved for the Ph.D. degree.

NOTATION. If $x \in E^{n}$, let

$$
S^{n}[x, \lambda]=\{y:|y-x|=\lambda\} \text { and } B^{n}[x, \lambda]=\{y:|y-x| \leqq \lambda\}
$$

denote the $n$-dimensional sphere and ball, respectively, of radius $\lambda$ and centre $x$.

For any $m$-dimensional polytope $P$, let $L(P), A^{m}(P)$ and $V^{m}(P)$ denote the total edgelength, surface area and volume of $P$, respectively. For a measurable $X$, let $\mu^{r}(X)$ denote the Hausdorff $r$-dimensional measure of $X$; see for example [2].

If $X, Y$ are convex sets, let $\delta(X, Y)$ denote the Hausdorff metric distance between $X$ and $Y$, see [3]. Let

$$
\rho(X, Y)=\inf _{\substack{x \in X \\ y \in Y}}|x-y| .
$$

Finally for an arbitrary set $X$, we shall write conv $X$ and aff $X$ to denote the convex hull and affine hull of $X$, respectively (so that aff $X$ is the linear subspace of $E^{n}$ of minimal dimension which contains $X$ ).

We shall first consider the case where all $r$-dimensional faces are restricted for $r=1, \ldots$, $n-1$. If $\varepsilon>0$, let $\mathscr{P}^{n, \lambda}(\varepsilon)$ denote the family of polytopes in $E^{n}$, which are inscribed in $S^{n}[o, \lambda]$, which contain $o$, and whose $r$-dimensional faces have $\mu^{r}$-measure less than $\varepsilon$ for $r=1, \ldots, n-1$.

LEMMA 1. Let $\delta^{n, \lambda}(\varepsilon)=\sup _{P \in \mathscr{G}^{n}, \lambda(\varepsilon)} \delta\left(P, B^{n}[0, \lambda]\right)$.

Then $\delta^{n, \lambda}(\varepsilon) \rightarrow 0$ as $\varepsilon \rightarrow 0$, if $n \geqq 2$.

Proof. The proof is by induction on the dimension $n$. Let $\delta$ be given with $0<\delta<\lambda$. Firstly suppose $n=2$. Then, if $\varepsilon<2\left(2 \lambda \delta-\delta^{2}\right)^{1 / 2}$, it follows that 


$$
\begin{aligned}
\delta\left(P, B^{2}[o, \lambda]\right) & <\lambda-\left[\lambda^{2}-(\varepsilon / 2)^{2}\right]^{1 / 2} \\
& <\delta
\end{aligned}
$$

whenever $P \in \mathscr{P}^{n, \lambda}(\varepsilon)$. The lemma then is proved when $n=2$ and we suppose the result is true in each dimension $k$ with $2 \leqq k \leqq n-1$.

Now each facet $F$ of $P$ is inscribed in an $(n-1)$-sphere which is the intersection of aff $F$ and $S^{n}[0, \lambda]$. Let $F^{\prime}$ denote that facet for which the corresponding $(n-1)$-sphere has maximal radius. Suppose this sphere has centre $o^{\prime}$ and radius $\mu$. We note that for every facet $F$

$$
\rho(o, F) \geqq \rho\left(o, F^{\prime}\right) \geqq \rho\left(o, \text { aff } F^{\prime}\right)=\rho\left(o, o^{\prime}\right) .
$$

Thus $o^{\prime} \in F^{\prime}$, for otherwise $o o^{\prime}$ would meet some facet in $o^{\prime \prime}$, with $o^{\prime \prime}$ not equal to $e^{\prime}$ and between $o$ and $o^{\prime}$. This would contradict (1). It is now convenient to write $P(\varepsilon)=P$, $o^{\prime}(\varepsilon)=o^{\prime}, F(\varepsilon)=F, F^{\prime}(\varepsilon)=F^{\prime}$ and $\mu(\varepsilon)=\mu$.

We shall show that there exists $\varepsilon_{0}(n, \lambda, \delta)$ such that

$$
\mu(\varepsilon)<\eta=\left[\lambda \delta-(\delta / 2)^{2}\right]^{1 / 2}
$$

whenever $P(\varepsilon) \in \mathscr{P}^{n, \lambda}(\varepsilon)$ and $\varepsilon<\varepsilon_{0}$. Suppose this is not so. Then there is a sequence $\left\{\varepsilon_{i}\right\}_{i=1}^{\infty}$ monotonically tending to zero as $i$ tends to infinity and a sequence of polytopes $P\left(\varepsilon_{i}\right) \in \mathscr{P}^{n, \lambda}\left(\varepsilon_{i}\right)$ such that

$$
\mu\left(\varepsilon_{i}\right) \geqq \eta \quad \text { for } \quad i=1,2, \ldots .
$$

Let $F^{\prime \prime}\left(\varepsilon_{i}\right)$ denote the polytope similar to $F^{\prime}\left(\varepsilon_{i}\right)$ which is reduced in the ratio $\eta: \mu\left(\varepsilon_{i}\right)$ about $o^{\prime}\left(\varepsilon_{i}\right)$ as centre of similitude for $i=1,2, \ldots$ By an appropriate translation we may further assume that $F^{\prime \prime}\left(\varepsilon_{i}\right)$ is inscribed in an $(n-1)$-sphere $S^{n-1}[o, \eta]$, and contains $o$ for $i=1,2, \ldots$ Further the $r$-dimensional faces of $F^{\prime \prime}\left(\varepsilon_{i}\right)$ have $r$-measure less than

$$
\left(\frac{\eta}{\mu\left(\varepsilon_{i}\right)}\right)^{r} \varepsilon_{i} \leqq \varepsilon_{i}
$$

for $r=1, \ldots n-2$ and $i=1,2, \ldots$ Thus

$$
F^{\prime \prime}\left(\varepsilon_{i}\right) \in \mathscr{P}^{n-1, \eta}\left(\varepsilon_{i}\right) \text { for } i=1,2, \ldots
$$

Hence by the induction hypothesis,

$$
\delta\left(F^{\prime \prime}\left(\varepsilon_{i}\right), B^{n-1}[0, \eta]\right) \rightarrow 0 \text { as } i \rightarrow \infty .
$$

But by the hypothesis of the lemma,

$$
V^{n-1}\left(F^{\prime \prime}\left(\varepsilon_{i}\right)\right) \rightarrow 0 \text { as } i \rightarrow \infty .
$$

It is well known, see for example [3], that $V^{n-1}$ is continuous over the metric $\delta$ and therefore (5) and (6) are contradictory. Thus statement (2) holds true.

Then if $\varepsilon<\varepsilon_{0}$, it follows that each facet of $P(\varepsilon)$ is distant at least $\left(\lambda^{2}-\eta^{2}\right)^{1 / 2}$ from $o$ and thus

$$
B\left[o,\left(\lambda^{2}-\eta^{2}\right)^{1 / 2}\right] \subset P(\varepsilon) \subset B[o, \lambda] .
$$


Hence for all $P(\varepsilon) \in \mathscr{P}^{n, \lambda}(\varepsilon)$,

$$
\begin{aligned}
\delta\left(P(\varepsilon), B^{n}[o, \lambda]\right) & \leqq \delta\left(B\left[o,\left(\lambda^{2}-\eta^{2}\right)^{1 / 2}\right], B[o, \lambda]\right) \\
& =\lambda-\left(\lambda^{2}-\eta^{2}\right)^{1 / 2} \\
& =\frac{\delta}{2} \text { by }(2) .
\end{aligned}
$$

Thus

and the lemma is proved.

$$
\delta^{n, \lambda}(\varepsilon)<\delta
$$

THEOREM 1. Let $A^{n, \lambda}(\varepsilon)=\inf _{P \in \mathscr{F}^{n, \lambda}(\varepsilon)} A^{n}(P)$.

Then

$$
A^{n, \lambda}(\varepsilon) \rightarrow A\left(B^{n}[o, \lambda]\right) \text { as } \varepsilon \rightarrow 0 \quad \text { if } n \geqq 2 \text {. }
$$

Proof. Let $\delta>0$ be given. For each $\varepsilon>0$, choose $P(\varepsilon) \in \mathscr{P}^{n, \lambda}(\varepsilon)$ satisfying

$$
A^{n, \lambda}(\varepsilon)+\frac{\delta}{2} \geqq A^{n}(P(\varepsilon)) \text {. }
$$

By Lemma 1 , if $\varepsilon<\varepsilon_{0}(n, \lambda, \delta)$, then

$$
A^{n}\left(B^{n}[0, \lambda]\right)-A^{n}(P(\varepsilon)) \leqq \frac{\delta}{2}
$$

since $A^{n}$ is continuous over the class of compact convex sets. Hence (9) and (10) imply

$$
A^{n, \lambda}(\varepsilon) \geqq A^{n}\left(B^{n}[o, \lambda]\right)-\delta .
$$

The remainder of the proof is trivial and is omitted.

LemMA 2. Let $X$ be a bounded set in $E^{n}, n \geqq 2$, with positive Lebesgue measure. Then if $\varepsilon>0$ and $\left\{x_{1}, \ldots, x_{q}\right\}$ is a finite set of points of $X$ with the property that

$$
\sup _{x \in X}\left(\min _{1 \leqq i \leqq q}\left|x-x_{i}\right|\right)<\varepsilon,
$$

then there exists a constant $M(n, X)$ such that all arc-wise connected sets $E$ containing $\left\{x_{1}, \ldots, x_{q}\right\}$ have linear measure $\mu^{1}(E)>M(n, X) \varepsilon^{-(n-1)}$.

Proof. Let $C$ be a closed hypercube containing $X$ of side-length $l$. For each integer $k$ divide $C$ into $(3 k)^{n}$ open disjoint equal hypercubes of side $l / 3 k$. Suppose exactly $m(k)$ of these hypercubes contain at least one point of $X$. Then $X$ is contained in the union of these $m(k)$ hypercubes together with a closed set $Y$ of Lebesgue measure zero. Thus

$$
m(k)\left(\frac{l}{3 k}\right)^{n} \geqq \lambda(X),
$$

where $\lambda(X)$ denotes the Lebesgue measure of $X$. 
Consider the partition of $C$ as an array of $(3 k)^{n}$ open hypercubes $C\left(i_{1}, \ldots, i_{n}\right)$ where $1 \leqq i_{j} \leqq 3 k$ for $j=1, \ldots, n$. Let

$$
T\left(p_{1}, \ldots, p_{n}\right)=\bigcup_{\substack{0 \leqq k-1 \\ 1}} C\left(3 r_{1}+p_{1}, \ldots, 3 r_{n}+p_{n}\right)
$$

where $1 \leqq p_{j} \leqq 3$ for $j=1, \ldots, n$. Then each $T\left(p_{1}, \ldots, p_{n}\right)$ is a union of open hypercubes and each is distant $2 l / 3 k$ from any other. Also

$$
C=\bigcup_{\substack{1 \leqq p_{i} \leqq 3 \\ 1 \leqq i \leqq n}} T\left(p_{1}, \ldots, p_{n}\right)
$$

and so if exactly $m\left(p_{1}, \ldots, p_{n}\right)$ hypercubes of $T\left(p_{1}, \ldots, p_{n}\right)$ contain at least one point of $X$ then

$$
\sum_{p_{1}=1}^{3} \ldots \sum_{p_{n}=1}^{3} m\left(p_{1}, \ldots, p_{n}\right)=m(k) \geqq\left(\frac{3 k}{l}\right) \lambda(X)
$$

by equation (12). Hence by equation (13), there exist particular values of $p_{1}, \ldots, p_{n}$ with $1 \leqq p_{1} \leqq 3, \ldots, 1 \leqq p_{n} \leqq 3$ for which

For these values we write

$$
m\left(p_{1}, \ldots, p_{n}\right) \geqq\left(\frac{k}{l}\right)^{n} \lambda(X) .
$$

$$
T\left(p_{1}, \ldots, p_{n}\right)=T \text { and } m\left(p_{1}, \ldots, p_{n}\right)=m .
$$

We may suppose, without loss of generality, that

$$
0<\varepsilon<l / 12 \text {. }
$$

We may further suppose $k$ so large that

$$
\frac{l}{6(k+1)}<\varepsilon \leqq \frac{l}{6 k}
$$

Denote the hypercubes of $T$ which contain a point of $X$ by $C_{1}, \ldots, C_{m}$. For $i=1,2, \ldots, m$, let $C_{i}^{\prime}$ and $C_{i}^{\prime \prime}$ be the hypercubes obtained from $C_{i}$ by magnifications of $C_{i}$ in the ratio $2: 1$ and 3:1 respectively about the centre of $C_{i}$ as centre of similitude. Now each $C_{i}$ contains a point of $X$, and by the choice of $\varepsilon$ it follows that any set $\left\{x_{1}, \ldots, x_{q}\right\}$ satisfying the condition of the lemma must have points in common with $C_{i}^{\prime}$ for $i=1, \ldots, m$. Then since $E$ is arc-wise connected it follows that there is an arc in $E$ joining each such point to the boundary of $C_{i}^{\prime \prime}$ which must therefore have length at least $l / 6 k$ for $i=1, \ldots, m$. Thus

$$
\begin{aligned}
\mu^{1}(E) & \geqq \frac{m l}{6 k} \lambda(X) \\
& \geqq \frac{1}{6} \cdot\left(\frac{k}{l}\right)^{n-1} \lambda(X) \text { by }(14) \\
& >M(n, X) \varepsilon^{-(n-1)} \text { by }(16) \text { and }(17),
\end{aligned}
$$

where $M(n, X)=2 \lambda(X) / 12^{n}$. The lemma is proved. 
THEOREM 2. Let $L^{n, \lambda}(\varepsilon)=\inf _{P \in \mathscr{P}^{n}, \lambda(\varepsilon)} L(P)$.

Then

$$
L^{n, \lambda}(\varepsilon) \rightarrow \infty \quad \text { as } \quad \varepsilon \rightarrow 0 \text { if } n \geqq 3 .
$$

Proof. Let $P(\varepsilon) \in \mathscr{P}^{n, \lambda}(\varepsilon)$ have vertices $x_{1}(\varepsilon), \ldots x_{m(\varepsilon)}(\varepsilon)$ and suppose

$$
L(P(\varepsilon)) \leqq L^{n, \lambda}(\varepsilon)+1 \text { for each } \varepsilon>0 .
$$

By the compactness of $S^{n}[o, \lambda]$ we can choose $x(\varepsilon)$ such that

and we may suppose

$$
\min _{1 \leqq i \leqq m(\varepsilon)}\left|x(\varepsilon)-x_{i}(\varepsilon)\right|=\sup _{x \in S^{n}[o, \lambda]} \min _{1 \leqq i \leqq m(\varepsilon)}\left|x-x_{i}(\varepsilon)\right|
$$

$$
\left|x(\varepsilon)-x_{1}(\varepsilon)\right|=\min _{1 \leqq i \leqq m(\varepsilon)}\left|x(\varepsilon)-x_{i}(\varepsilon)\right|
$$

for each $\varepsilon>0$.

We shall now show

$$
\left|x(\varepsilon)-x_{1}(\varepsilon)\right| \rightarrow 0 \text { as } \varepsilon \rightarrow 0 .
$$

Otherwise there exists $\delta>0$ and a sequence $\left\{\varepsilon_{j}\right\}_{j=1}^{\infty}$ tending to zero as $j$ tends to infinity such that

$$
\left|x\left(\varepsilon_{j}\right)-x_{1}\left(\varepsilon_{j}\right)\right| \geqq \delta \text { for } j=1,2, \ldots
$$

But, for $j=1,2, \ldots$,

$$
\begin{aligned}
\rho\left(P\left(\varepsilon_{j}\right), x\left(\varepsilon_{j}\right)\right) & \geqq \frac{\left|x\left(\varepsilon_{j}\right)-x_{1}\left(\varepsilon_{j}\right)\right|^{2}}{2 \lambda} \\
& \geqq \frac{\delta^{2}}{2 \lambda} \quad \text { by }(21) .
\end{aligned}
$$

But by Lemma 1,

$$
\delta\left(P\left(\varepsilon_{j}\right), B^{n}[o, \lambda]\right) \rightarrow 0 \quad \text { as } j \rightarrow \infty,
$$

and thus (22) and (21) are contradictory. Thus statement (20) follows and we may choose a sequence $\delta(\varepsilon)$ tending to zero as $\varepsilon$ tends to zero such that

$$
\sup _{x \in S^{n}[o, \lambda]} \min _{1 \leqq i \leqq m(\varepsilon)}\left|x-x_{i}(\varepsilon)\right|<\delta(\varepsilon)
$$

for each $\varepsilon>0$.

We next project by orthogonal projection on to an $(n-1)$-dimensional subspace of $E^{n}$ which contains $o$. Then the union of the edges of $P(\varepsilon)$ projects on to an arc-wise connected set $E$ say, which contains the projections of the vertices of $P(\varepsilon)$. Then by application of Lemma 2 with the projection of $S^{n}[o, \lambda]$ in place of $X$ and noting that distances are not increased under orthogonal projection, it follows that there exists a constant $M(n, \lambda)$ for which

$$
L(P(\varepsilon)) \geqq \mu^{1}(\varepsilon)>M(n, \lambda) \delta(\varepsilon)^{-(n-2)} .
$$


Thus

$$
L^{n, \lambda}(\varepsilon) \geqq M(n, \lambda) \delta(\varepsilon)^{-(n-2)}-1
$$

for each $\varepsilon>0$ by (18), and the theorem is proved.

We note that the lower bounds for both the surface area and total edge-length of polytopes in the class $\mathscr{P}^{n, 2}(\varepsilon)$ are indeed greater than in the general case; for example, by considering the extremal simplex described in [1]. The conditions imposed on the polytopes in the previous theorems seem rather severe in that the measures of all the non-trivial faces are restricted. However, again by considering the example above, it is possible to restrict all faces of dimension greater than 1 and yet still not be able to improve the bounds of zero for the sum of the measures of the $r$-dimensional faces for $2 \leqq r \leqq n-1$ and $2 n \lambda$, the conjectured lower bound for the total edge-lengths in the general case.

It is possible, however, to obtain different and interesting results by restricting only the edges and relaxing the conditions on the remaining faces. We illustrate this with two examples in $E^{3}$.

If $\varepsilon>0$, let $\mathscr{P}^{3, \lambda}(\varepsilon)$ denote the family of polyhedra in $E^{3}$, which are inscribed in $S^{3}[o, \lambda]$, which contains $o$, and whose edges have length less than $\varepsilon$.

THEOREM 3. Let $\bar{A}^{3, \lambda}(\varepsilon)=\inf _{P \in \overline{\mathscr{B}}^{3, \lambda} \lambda(\varepsilon)} A(P)$.

Then

$$
\bar{A}^{3, \lambda}(\varepsilon) \rightarrow 2 \pi \lambda^{2} \text { as } \varepsilon \rightarrow 0 .
$$

Proof. We shall first prove that

$$
\lim _{\varepsilon \rightarrow 0} \bar{A}^{3, \lambda}(\varepsilon) \leqq 2 \pi \lambda^{2}
$$

by an appropriate example.

For each positive integer $n$ let $P_{n}$ be a regular polygon of $n$ sides which is inscribed in $S^{3}[o, \lambda]$ and distant $\lambda / n^{2}$ from $o$ and let $P_{n}^{\prime}$ denote the reflection of $P_{n}$ in $o$.

Then the polyhedron $Q_{n}=\operatorname{conv}\left(P_{n}, P_{n}^{\prime}\right)$ has surface area

$$
\begin{aligned}
A\left(Q_{n}\right) & =\hat{\lambda}^{2} n\left(1-1 / n^{4}\right) \sin (2 \pi / n)+\left(4 \lambda^{2} / n\right)\left(1-1 / n^{4}\right)^{1 / 2} \sin (\pi / n) \\
& \rightarrow 2 \pi \lambda^{2} \text { as } n \rightarrow \infty .
\end{aligned}
$$

Thus (25) follows.

It remains to prove the reverse inequality. Let $\delta$ be given with $\lambda>\delta>0$ and consider $\varepsilon>0$ satisfying

$$
0<\frac{\varepsilon^{2}}{4}<2 \delta \lambda-\delta^{2}
$$

Let $P \in \mathscr{P}^{3, \lambda}$ and suppose $F$ is any facet of $P$. Then aff $F$ either does not meet $D^{3}[0, \lambda-\delta]$ or meets $D^{3}[o, \lambda-\delta]$ in a single point or in a disc $D^{2}[u, \eta]$, say. Now if the latter case occurs, suppose aff $F$ meets $D^{3}[0, \lambda-\delta]$ in the disc $D^{n}[u, v]$ where $v>\eta$. Then each edge of $F$ is 
distant at least

from $u$.

$$
\begin{aligned}
\left(v^{2}-\frac{\varepsilon^{2}}{4}\right)^{1 / 2} & >\left(v^{2}-2 \delta \lambda+\delta^{2}\right)^{1 / 2} \text { by } \\
& =\left((\lambda-\delta)^{2}-\left(\lambda^{2}-v^{2}\right)\right)^{1 / 2} \\
& =\eta
\end{aligned}
$$

Thus each facet of $P$ either does not meet $D^{3}[0, \lambda-\delta]$ or meets $D^{3}[0, \lambda-\delta]$ in a single point or in a disc. Suppose there are $N$ such discs and that each is distant $\lambda_{t}$ from 0 for $i=1, \ldots, N$. Also, let $\bar{\lambda}=\lambda-\delta$. Then

$$
A(P) \geqq 4 \pi \pi^{2}-A^{\prime}(P)+A^{\prime \prime}(P)
$$

where $A^{\prime}(P)$ is the area of the surface of $D^{3}[o, \lambda]$ which lies exterior to $P$, and $A^{\prime \prime}(P)$ is the total surface area of the discs.

We note

$$
A^{\prime}(P)=\sum_{i=1}^{N} 2 \pi \lambda\left(\bar{\lambda}-\lambda_{i}\right)
$$

and

$$
A^{\prime \prime}(P)=\sum_{i=1}^{N} \pi\left(\bar{\lambda}^{2}-\lambda_{i}^{2}\right) .
$$

Now trivially $4 \pi \lambda^{2} \geqq A^{\prime}(P)$, and so if $A^{\prime \prime}(P) \geqq 2 \pi \lambda^{2}$, then $A(P) \geqq 2 \pi \lambda^{2}$ by (28). Otherwise suppose

Then (28), (29), (30) imply

$$
A^{\prime \prime}(P)<2 \pi \bar{\lambda}^{2}
$$

$$
\begin{aligned}
A(P) & \geqq 4 \pi \bar{\lambda}^{2}-\sum_{i=1}^{N} \pi\left(\bar{\lambda}-\lambda_{i}\right)^{2} \\
& \geqq 4 \pi \bar{\lambda}^{2}-\sum_{i=1}^{N} \pi\left(\bar{\lambda}^{2}-\lambda_{i}^{2}\right), \text { since } \bar{\lambda} \geqq \lambda_{i} \text { for } i=1, \ldots, N, \\
& =4 \pi \bar{\lambda}^{2}-A^{\prime \prime}(P) \\
& >2 \pi \bar{\lambda}^{2} \text { by }(31) .
\end{aligned}
$$

Hence

$$
\bar{A}^{3, \lambda}(\varepsilon) \geqq 2 \pi \bar{\lambda}^{2}=2 \pi(\lambda-\delta)^{2},
$$

and the theorem follows since the choice of $\delta$ was arbitrary.

LEMMA 3. Let $X$ be a subset of $S^{3}[0, \lambda]$ of positive $\mu^{2}$-measure. Then the orthogonal projection of $X$ on to any plane has positive $\mu^{2}$-measure.

The proof involves standard arguments in measure theory and in order to save space is omitted.

THEOREM 4. Let $\bar{L}^{3, \lambda}(\varepsilon)=\inf _{P \in \overline{9}^{3}, \lambda(\varepsilon)} L(P)$.

Then

$$
L^{3, \lambda}(\varepsilon) \rightarrow 4 \pi \lambda \text { as } \varepsilon \rightarrow 0
$$


Proof. The polyhedron $Q_{n}$ defined in the beginning of Theorem 3 has total edge-length

$$
\begin{aligned}
L\left(Q_{n}\right) & =4 \lambda n\left(1-1 / n^{4}\right)^{1 / 2} \sin (\pi / n)+2 \lambda / n \\
& \rightarrow 4 \pi \lambda \text { as } n \rightarrow \infty .
\end{aligned}
$$

Thus

$$
\lim _{\varepsilon \rightarrow 0} L^{3, \lambda}(\varepsilon) \leqq 4 \pi \lambda
$$

Let $\delta>0$ be given. In each $\varepsilon>0$, choose $P(\varepsilon) \in \mathscr{P}^{3, \lambda}$ so that

$$
L(P(\varepsilon)) \leqq L^{3, \lambda}(\varepsilon)+\delta .
$$

Then by the Blaschke selection theorem, we may assume that there exists a sequence $\left\{\varepsilon_{i}\right\}_{i=1}^{\infty}$ tending to zero as $i$ tends to infinity such that $P\left(\varepsilon_{i}\right)$ tends to a convex set $P$ contained in $D^{3}[o, \lambda]$ and also such that $L\left(P\left(\varepsilon_{i}\right)\right)$ tends to some number $L$ as $i$ tends to infinity. Further, by (33) and (32),

$$
L \leqq 4 \pi \lambda+\delta .
$$

Now it was shown in Theorem 3 that, if $0<\eta<\lambda$, then either $D^{3}[0, \lambda-\eta] \subset P\left(\varepsilon_{i}\right)$ or $D^{3}[o, \lambda-\eta]$ meets the frontier of $P\left(\varepsilon_{i}\right)$ in closed discs, whenever $i$ is so large that

$$
\varepsilon_{i}^{2} / 4<2 \eta \lambda-\eta^{2}
$$

Hence, taking the limit as $i$ tends to infinity, it follows that $P$ has this property also. Now there exists $\eta_{0}>0$ for which $D^{3}[o, \lambda-\eta]$ meets fr $P$, the frontier of $P$ in at least one disc. Otherwise $P \subset D^{3}[o, \lambda-\eta]$ for each $\eta>0$. This implies that $P\left(\varepsilon_{i}\right)$ tends to $D^{3}[o, \lambda]$ as $i \rightarrow \infty$ and we could show, by an argument similar to that given in Theorem 2 , that

$$
L\left(P\left(\varepsilon_{i}\right)\right) \rightarrow \infty \text { as } i \rightarrow \infty .
$$

This would contradict (34).

We shall show that for each $\eta, 0<\eta<\eta_{0}, D^{3}[o, \lambda-\eta]$ meets fr $P$ in a finite number of discs (of positive radius). Now if $i$ is sufficiently large, then again, as in Theorem 3, we may suppose that fr $P\left(\varepsilon_{i}\right)$ only meets $D^{3}[o, \lambda-\eta]$ in $m_{i}>0$ discs. Further each such facet of $P\left(\varepsilon_{i}\right)$ which meets $D^{3}[0, \lambda-\eta]$ is inscribed in a circle of radius at least $\left(\lambda^{2}-(\lambda-\eta)^{2}\right)^{1 / 2}$. Thus if $\ddot{\xi}$ is given, $0<\xi<\left(\lambda^{2}-(\lambda-\eta)^{2}\right)^{1 / 2}$, then, by Theorem 1 , all $m_{i}$ facets of $P\left(\varepsilon_{i}\right)$ which meet $D^{3}[o, \lambda-\eta]$ contain a disc of radius at least $\left(\lambda^{2}-(\lambda-\eta)^{2}\right)^{1 / 2}-\xi$ whenever $i$ is sufficiently large. But, since $P\left(\varepsilon_{i}\right) \subset D^{3}[o, \lambda]$,

$$
4 \pi \lambda^{2} \geqq m_{i} \pi\left[\left(\lambda^{2}-(\lambda-\eta)^{2}\right)^{1 / 2}-\xi\right]^{2},
$$

and so for given $\eta$ the sequence $\left\{m_{i}\right\}_{i=1}^{\infty}$ is bounded.

We shall now then consider $\eta<\eta_{0}$, and so, for all $i$ sufficiently large, fr $P\left(\varepsilon_{i}\right)$ will meet $D^{3}[o, \lambda-\eta]$ in $m_{i}>0$ discs. Let $F_{1}\left(\varepsilon_{i}\right), \ldots, F_{m_{i}}\left(\varepsilon_{i}\right)$ denote the $m_{i}$ facets of $P\left(\varepsilon_{i}\right)$ which meet $D^{3}[0, \lambda-\eta]$. Let $L^{\prime}\left(\varepsilon_{i}\right)$ denote the sum of the edges of the polygons $F_{1}\left(\varepsilon_{i}\right), \ldots, F_{m i}\left(\varepsilon_{i}\right)$ for $i=1,2, \ldots$.

Then, since any two of these polygons have at most one side in common with each other, 
it follows that

$$
L^{\prime}\left(\varepsilon_{i}\right)+m_{i}^{m_{i}} \varepsilon_{i} \geqq \sum_{j=1}^{m_{i}} L\left(F_{j}\left(\varepsilon_{i}\right)\right)
$$

for $i=1,2, \ldots$. Let $L^{\prime \prime}\left(\varepsilon_{i}\right)$ be the sum of the circumferences of the discs formed by the intersection of $D^{3}[0, \lambda-\eta]$ with fr $P\left(\varepsilon_{t}\right)$. Let $L^{\prime \prime}$ denote the corresponding sum for

Then

$$
D^{3}[0, \lambda-\eta] \cap \text { fr } P \text {. }
$$

Also trivially

$$
\sum_{j=1}^{m_{4}} L\left(F_{j}\left(\varepsilon_{i}\right)\right) \geqq L^{\prime \prime}\left(\varepsilon_{i}\right) \text { for } \quad i=1,2, \ldots
$$

$$
L\left(P\left(\varepsilon_{i}\right)\right) \geqq L^{\prime}\left(\varepsilon_{i}\right) \quad \text { for } \quad i=1,2, \ldots
$$

Thus (36), (37) and (38) imply

$$
L\left(P\left(\varepsilon_{i}\right)\right)+m_{i}^{m_{i}} \varepsilon_{i} \geqq L^{\prime \prime}\left(\varepsilon_{i}\right) \text { for } i=1,2, \ldots
$$

Since $P\left(\varepsilon_{i}\right)$ tends to $P$ as $i$ tends to infinity it follows that $D^{3}[0, \lambda-\eta) \cap P\left(\varepsilon_{i}\right)$ tends to $D^{3}[0, \lambda-\eta] \cap P$ as $i$ tends to infinity. Thus

$$
L^{\prime \prime}\left(\varepsilon_{i}\right) \rightarrow L^{\prime \prime} \text { as } i \rightarrow \infty .
$$

Hence, since the sequence $\left\{m_{i}\right\}_{i=1}^{\infty}$ is bounded, it follows from (39) that

$$
L \geqq L^{\prime \prime} \text {. }
$$

Now consider a sequence $\left\{\eta_{j}\right\}_{j=1}^{\infty}$ tending to zero monotonically as $j$ tends to infinity with $\eta_{j}<\eta_{0}$ for $j=1,2, \ldots$ Then we can assume $D^{3}\left[o, \lambda-\eta_{j}\right]$ meets fr $P$ in $l_{j}$ disjoint discs $D_{1}\left(\eta_{j}\right), \ldots, D_{l_{j}}\left(\eta_{j}\right)$ of radii $\lambda_{1}\left(\eta_{j}\right), \ldots, \lambda_{l}\left(\eta_{j}\right)$ respectively and so $\operatorname{fr}\left(D^{3}\left[o, \lambda-\eta_{j}\right] \cap P\right)$ consists of these discs together with an open subset $W\left(\eta_{j}\right)$ of $S^{3}\left[o, \lambda-\eta_{j}\right]$ for $j=1,2, \ldots$ Let $D_{k}^{\prime}\left(\eta_{j}\right)$ denote the projection of the disc $D_{k}\left(\eta_{j}\right)$ from $o$ on to $S^{3}[o, \lambda]$ for $k=1, \ldots, l_{j}$, and similarly $W^{\prime}\left(\eta_{j}\right)$ as the projection of $W\left(\eta_{j}\right)$ for $j=1,2, \ldots$.

The $D_{k}^{\prime}\left(\eta_{j}\right)$ are "caps" of $S^{3}[0, \lambda]$ with a base of radius $\lambda_{k}^{\prime}\left(\eta_{j}\right)$ say, where

$$
\lambda_{k}^{\prime}\left(\eta_{j}\right)=\frac{\lambda}{\lambda-\eta_{j}} \lambda_{k}\left(\eta_{j}\right)
$$

for $k=1, \ldots, l_{j}$ and $j=1,2, \ldots$ We note also that

$$
D_{k}^{\prime}\left(\eta_{j+1}\right) \supset D_{k}^{\prime}\left(\eta_{j}\right) \text { and } W^{\prime}\left(\eta_{j+1}\right) \subset W^{\prime}\left(\eta_{j}\right)
$$

for $k=1, \ldots, l_{j}$, and $j=1,2, \ldots$.

Now equation (40) implies

$$
\begin{aligned}
L & \geqq 2 \pi \sum_{k=1}^{l_{j}} \lambda_{k}\left(\eta_{j}\right) \\
& =(2 \pi / \lambda)\left(\lambda-\eta_{j}\right) \sum_{k=1}^{l_{j}} \lambda_{k}^{\prime}\left(\eta_{j}\right) \\
& \rightarrow 2 \pi \sum_{k=1}^{l} \lambda_{k}^{\prime} \text { as } j \rightarrow \infty
\end{aligned}
$$


where we write

and

$$
\lambda_{k}^{\prime}=\lim _{j \rightarrow \infty} \lambda_{k}^{\prime}\left(\eta_{j}\right) \text { for } k=1, \ldots, l
$$

$$
l=\lim _{j \rightarrow \infty} l_{j}
$$

We note that these limits exist since both sequences are monotonically increasing with $j$. The possibility that $l=\infty$ is not excluded. Now

$$
4 \pi \lambda^{2}=\frac{\pi}{4} \mu^{2}\left(W^{\prime}\left(\eta_{j}\right)\right)+2 \pi \sum_{k=1}^{l_{j}} \lambda\left[\lambda-\left(\lambda^{2}-\lambda_{k}^{\prime}\left(\eta_{j}\right)^{2}\right)^{1 / 2}\right]
$$

where we have used the fact that the area of the curved surface of a " cap " is equal to $4 / \pi$ times its Hausdorff 2-dimensional measure. This follows from a result on page 54 of [2].

Further, (42) implies that

$$
\mu^{2}\left(\bigcap_{j=1}^{l} W^{\prime}\left(\eta_{i}\right)\right)=\lim _{j \rightarrow \infty} \mu^{2}\left(W^{\prime}\left(\eta_{i}\right)\right)
$$

and thus, taking the limit as $j$ tends to infinity in (44), we obtain

$$
4 \pi \lambda^{2}=\mu^{2}\left(\bigcap_{j=1}^{l} W^{\prime}\left(\eta_{i}\right)\right)+2 \pi \sum_{k=1}^{l} \lambda\left(\lambda-\left(\lambda^{2}-\lambda_{k}^{\prime 2}\right)^{1 / 2}\right) .
$$

Suppose firstly that

$$
\mu^{2}\left(\bigcap_{j=1}^{l} W^{\prime}\left(\eta_{i}\right)\right)>0
$$

Then, if $P\left(\varepsilon_{i}\right)$ has vertices $v_{1}\left(\varepsilon_{i}\right), \ldots, v_{n}\left(\varepsilon_{i}\right)$, it is not difficult to show that

$$
\left.\sup _{\substack{w \in \prod_{j=1}^{\infty} W^{\prime}\left(\eta_{j}\right) \\ j \leqq k \leqq v_{n_{i}}}}\left|w-v_{k}\left(\varepsilon_{i}\right)\right|\right) \rightarrow 0 \text { as } \varepsilon \rightarrow 0 \text {. }
$$

We now project $E^{3}$ by orthogonal projection on to a plane. By Lemma $3, \bigcap_{j=1}^{\infty} W^{\prime}\left(\eta_{j}\right)$ projects on to a set of positive $\mu^{2}$-measure and thus positive Lebesgue measure. Then, using the argument of Theorem 2 it is not difficult to show that this implies

$$
L\left(P\left(\varepsilon_{i}\right)\right) \rightarrow \infty \text { as } i \rightarrow \infty .
$$

This would contradict equation (34). Thus

and so, from (46),

$$
\mu^{2}\left(\bigcap_{j=1}^{\infty} W^{\prime}\left(\eta_{j}\right)\right)=0
$$

$$
\begin{aligned}
4 \pi \lambda^{2} & =2 \pi \sum_{k=1}^{l} \lambda\left(\lambda-\left(\lambda^{2}-\lambda_{k}^{\prime 2}\right)^{1 / 2}\right) \\
& \leqq 2 \pi \lambda \sum_{k=1}^{l} \lambda_{k}^{\prime} .
\end{aligned}
$$


Thus (43) and (50) imply

and so, by (33),

$$
L \geqq 4 \pi \lambda
$$

$$
\delta+\lim _{\varepsilon \rightarrow 0} L^{3, \lambda}(\varepsilon) \geqq 4 \pi \lambda .
$$

Since the choice of $\delta$ was arbitrary, the theorem is proved.

REMARKS. It is interesting to look at some possible generalisations of these theorems for arbitrary convex sets. For example, suppose $X$ is an arbitrary convex set with a nonempty interior int $X$, with $x \in$ int $X$ and that $\mathscr{P}^{n, \lambda}(X, x ; \varepsilon)$ is the family of polytopes which are inscribed in $X$ (i.e. the vertices of each polytope are contained in the frontier of $X$ ), which contain $x$, and whose $r$-dimensional faces have $\mu^{r}$-measure less than $\varepsilon$ for $r=1, \ldots$, $n-1$. We can then show that if

$$
\delta^{n, \lambda}(X, x ; \varepsilon)=\sup _{P_{E} \operatorname{gnn}_{\lambda} \lambda(X, x ; \varepsilon)}(P, X)
$$

then $\delta^{n, \lambda}(X, x ; \varepsilon)$ tends to zero as $\varepsilon$ tends to zero, whenever $n \geqq 2$. The proof of Lemma 1 readily generalises, since, by using an argument similar to that described, we can show that the radii of the circumspheres of each facet of $P(\varepsilon)$ tend to zero as $\varepsilon$ tends to zero. We can then prove the natural extensions of Theorems 1 and 2 with $\mathscr{P}^{n, \lambda}(X, x ; \varepsilon)$ in place of $\mathscr{P}^{n, \lambda}(\varepsilon)$ by the same methods as those described in these theorems.

There are more interesting conjectures concerned with Theorems 3 and 4 . For example if $\mathscr{P}^{3}, \lambda(X, x ; \varepsilon)$ is the family of polyhedra inscribed in $X$, which contain $x$, and whose edges have length less than $\varepsilon$, then we might hope to prove:

CONJECTURE. Let

Then

$$
\left\{\begin{array}{l}
A^{3, \lambda}(X, x ; \varepsilon) \\
L^{3, \lambda}(X, x ; \varepsilon)
\end{array}\right\}=\inf _{P \in \overline{\mathcal{P}}^{n}, \lambda(X, x ; \varepsilon)}\left\{\begin{array}{l}
A^{3}(P) \\
L(P)
\end{array}\right\} .
$$

$$
\left\{\begin{array}{l}
A^{3, \lambda}(X, x ; \varepsilon) \\
L^{3, \lambda}(X, x ; \varepsilon)
\end{array}\right\} \rightarrow 2 \inf _{x \in H}\left\{\begin{array}{l}
A^{2}(H \cap X) \\
P(H \cap X)
\end{array}\right\} \text { as } \varepsilon \rightarrow 0,
$$

where the infimum is over all planes $H$ containing $x$, and $P(H \cap X)$ is the perimeter of $H \cap X$.

It would also be interesting to have $n$-dimensional analogues of these theorems or to prove other general theorems associated with polytopes which have restricted edge-lengths.

\section{REFERENCES}

1. J. N. Lillington, A conjecture for polytopes, Proc. Cambridge Philos. Soc. 76 (1974), 407-411.

2. C. A. Rogers, Hausdorff measures (Cambridge, 1970).

3. H. G. Eggleston, Convexity (Cambridge, 1969).

\section{NoRThPoRt Drive WaReham, DORSET}

